\title{
Spinal cord stimulation for treatment of the pain associated with hereditary multiple osteochondromas
}

This article was published in the following Dove Press journal:

Journal of Pain Research

18 August 2015

Number of times this article has been viewed

\author{
Ravi G Mirpuri' \\ Jereme Brammeier ${ }^{2}$ \\ Hamilton Chen ${ }^{2}$ \\ Frank PK Hsu ${ }^{1,3}$ \\ Vi K Chiu ${ }^{4}$ \\ Eric Y Chang ${ }^{1,2,5}$
}

'Department of Physical Medicine and Rehabilitaiton, ${ }^{2}$ Department of Anesthesiology and Perioperative Care, ${ }^{3}$ Department of Neurological Surgery, ${ }^{4}$ Department of Medicine, ${ }^{5}$ Reeve-Irvine Research Center for Spinal Cord Injury, University of California Irvine, Irvine, CA, USA

Correspondence: Eric Y Chang Department of Physical Medicine and Rehabilitation, University of California Irvine, Gillespie Building Rm 3226A, 837 Health Science Road, Irvine, CA 92697. 1050, USA

Tel + I 9498247222

Email changeyl@uci.edu
Objective: Hereditary multiple osteochondromas (HMO) usually presents with neoplastic lesions throughout the skeletal system. These lesions frequently cause chronic pain and are conventionally treated with surgical resection and medication. In cases where conventional treatments have failed, spinal cord stimulation (SCS) could be considered as a potential option for pain relief. The objective of this case was to determine if SCS may have a role in treating pain secondary to neoplastic lesions from HMO.

Case presentation: We report a 65 -year-old female who previously received both surgical and pharmacological interventions for treating HMO neoplastic pain in the lumbar, pelvis, femur, and tibial regions. These interventions either failed to offer significant pain relief or caused excessive lethargy. A SCS trial was then offered with a dual 16-contact lead trial leading to $70 \%-80 \%$ improvement in pain from baseline and $85 \%$ reduction in oxycodone IR intake. This was followed by permanent implantation of two $2 \times 8$ contact paddle leads (T7-T8 and T9-T10 interspaces). After 8-week follow-up, settings were further optimized resulting in an additional $30 \%$ improvement in pain compared to last visit. At 6-month follow-up, the patient reported continued pain relief.

Conclusion: This case demonstrates the first successful use of SCS to treat both HMO and nonmalignant neoplastic-related pain. The patient reported pain improvement from baseline, reduced pain medication requirements, and subjective improvement in quality of life. Additionally, this case demonstrates the potential advantage of trialing multiple painful areas with a 16-contact lead in order to avoid multiple trials and placement.

Keywords: Exostoses, bone neoplasms/pathology, hereditary exostoses/multiple, back pain/ therapy, pain management, spinal cord stimulation, treatment outcome, chronic pain, case reports

\section{Introduction}

Hereditary multiple osteochondromas (HMO) is primarily an autosomal dominant disorder where benign osteochondromas form throughout the skeletal system. Common locations of formation are the metaphyses of long bones including the femur, tibia, and humerus. ${ }^{1}$ This rare disorder has a prevalence of one in 50,000 with nearly all patients diagnosed before the age of 12 years. ${ }^{2,3}$ Treatment is recommended when lesions become symptomatic with manifestations of pain, peripheral nerve impingement, or debilitating bone deformities. ${ }^{4}$ In most cases, surgical excision is the main treatment although recurrence rates are high, especially with incomplete resection or in rare cases of malignant transformation. ${ }^{5,6}$

Although historically HMO is never treated, spinal cord stimulation (SCS) has been shown to provide symptomatic pain relief for refractory anginal pain, ischemic 
pain secondary to peripheral vascular disease, peripheral neuropathic pain, failed back surgery syndrome, and complex regional pain syndrome. ${ }^{78} \mathrm{SCS}$ is postulated to work through multiple mechanisms including attenuation of dorsal horn neuronal hyperactivity, increased GABA neurotransmitter release, and activation of descending pain-controlling pathways. ${ }^{9,10}$

In a recent 2013 Cochrane Review, the benefits of SCS for neoplastic conditions were evaluated and determined inconclusive, despite several studies demonstrating significant pain relief. ${ }^{11}$ Currently, the effectiveness of SCS on neoplastic-mediated pain is still being researched and requires more evidence. We present a case where SCS was successfully used to treat pain from osteochondromas. To our knowledge, this is one of the first reports of nonmalignant bone pain successfully treated with SCS.

\section{Case presentation}

A 65-year-old female with a past medical history of HMO presented to the outpatient pain clinic with a 4-year history of back and bilateral leg pain. Patient consent was obtained and de-identified data was utilized for this case report. The pain was characterized as constant, heavy, and aching in nature with an intermittent stabbing component that radiated from the back into her lower extremities. Isolated aching pain was also identified in the left hip, bilateral knees, and bilateral ankles. Pain was assessed using a numeric pain scale of $0-10$ with 0 reflecting no pain, $1-3$ reflecting mild pain and mild interference on activities of daily living (ADL), 4-6 reflecting moderate pain and moderate interference on ADL, and 7-10 reflecting severe pain and inability to perform ADL. The patient initially reported an average pain of 5/10 and reaching a maximum pain of 9/10. Exacerbating factors included walking, exercise, spinal flexion, and rotation. On physical examination, the patient presented with multiple tender points in the lumbar paraspinals, hips, and ankles. There were palpable osteochondromas in the region of the distal tibias along with pretibial allodynia extending inferiorly to the medial malleoli bilaterally. Motor strength was $5 / 5$ in all extremities, and there was no noted sensory deficit.

Lumbar MRI demonstrated an L5-S1 posterior fusion (previously performed 3 years ago to treat suspected Bertolotti's syndrome), moderate L2-L3 facet arthritis, and no significant central canal or neural foraminal stenosis. The patient's prior fusion provided minimal axial back pain relief and no effect in the lower extremities making Bertolotti's syndrome an unlikely cause for the pain. Electrodiagnostic studies showed mild demyelination of the left common peroneal nerve across fibular head and no evidence of lumbar radiculopathy. Further lower extremity radiographs demonstrated left pelvic, right distal femur, right proximal tibia, and left proximal tibia osteochondromas. Surgical excision was then performed for all the osteochondromas with minimal resolution of symptoms. Follow-up X-rays were repeated approximately a year later and showed new bilateral distal tibia osteochondromas of the metadiaphsysis region. Further surgery was declined, and the patient received numerous interventional pain procedures for various presentations including lumbar epidural steroid injections, ischial bursa injections, and bilateral piriformis injections. The pain was pharmacologically managed with methadone $5 \mathrm{mg}$ three times daily, methocarbamol $750 \mathrm{mg}$ four times daily, and oxycodone immediate release with an average daily consumption of $30-35 \mathrm{mg}$ for breakthrough pain. Multiple neuropathic medications and higher doses of methadone were trialed in the past but discontinued secondary to excess sedation.

The patient subsequently was referred for a trial of SCS. Two 16-contact leads were percutaneously placed under fluoroscopy spanning the right and left of T7-T9 vertebral bodies (Figure 1). Settings for both leads were optimized at an amplitude of $5.2 \mathrm{~mA}$, pulse width of $210 \mu \mathrm{S}$, and frequency of $40 \mathrm{~Hz}$. The patient was discharged home for 5 days with the trial leads and reported $70 \%-80 \%$ improvement in pain and $85 \%$ reduction in oxycodone IR intake.

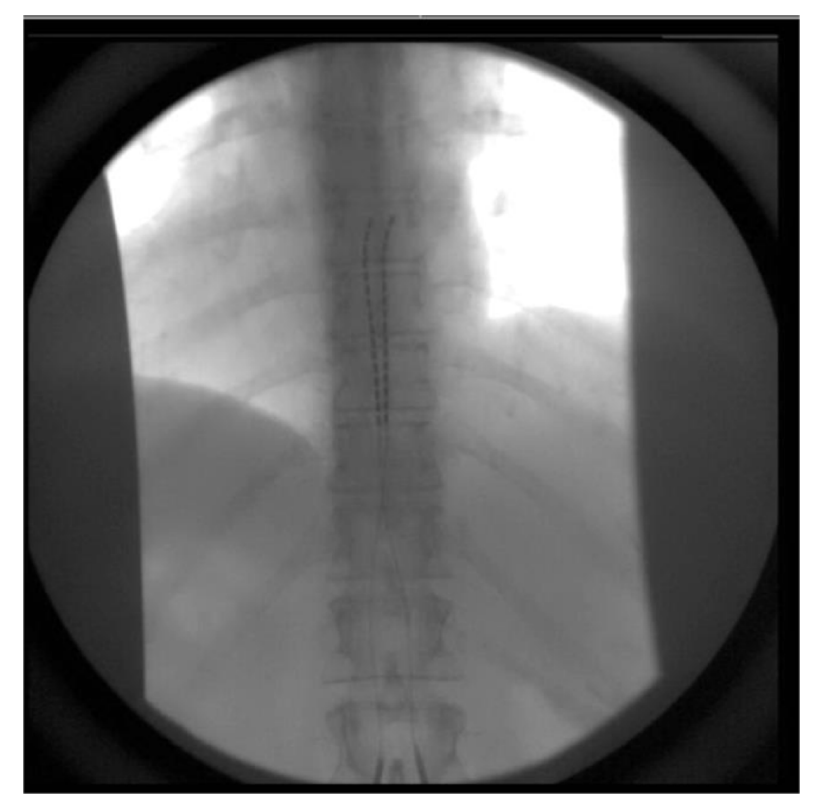

Figure I 16 contact lead spinal cord stimulator trial placed in the T8-T9 epidural space. 
The patient was then scheduled for permanent implantation with two $2 \times 8$ contact paddle leads and an implantable pulse generator that can accommodate 32 contacts. The patient was taken to the operating room under general anesthesia where a T8-T9 hemilaminectomy was performed and two longitudinal paddle leads were placed overlying areas of the T7-T8 and T9-T10 interspaces (Figure 2). The two paddle leads were utilized in order to cover pain from the back, hip, and ankles. The T7-T8 paddle lead was programmed to an amplitude of $2.6 \mathrm{~mA}$, pulse width of $290 \mu \mathrm{S}$, and frequency of $40 \mathrm{~Hz}$. The T9-T10 paddle lead was set to an amplitude of $4.1 \mathrm{~mA}$, pulse width of $470 \mu \mathrm{S}$, and frequency of $40 \mathrm{~Hz}$. On 8-week follow-up, settings were adjusted, and the patient reported another $30 \%$ reduction in pain compared to last visit's baseline, complete discontinuation of methadone, and increased ADL such as being able to climb steps more easily. At 6-month follow-up, the patient reported continued relief.

Written informed consent was obtained from our patient for publication of this case report and any accompanying images. Institutional Review Board was not needed

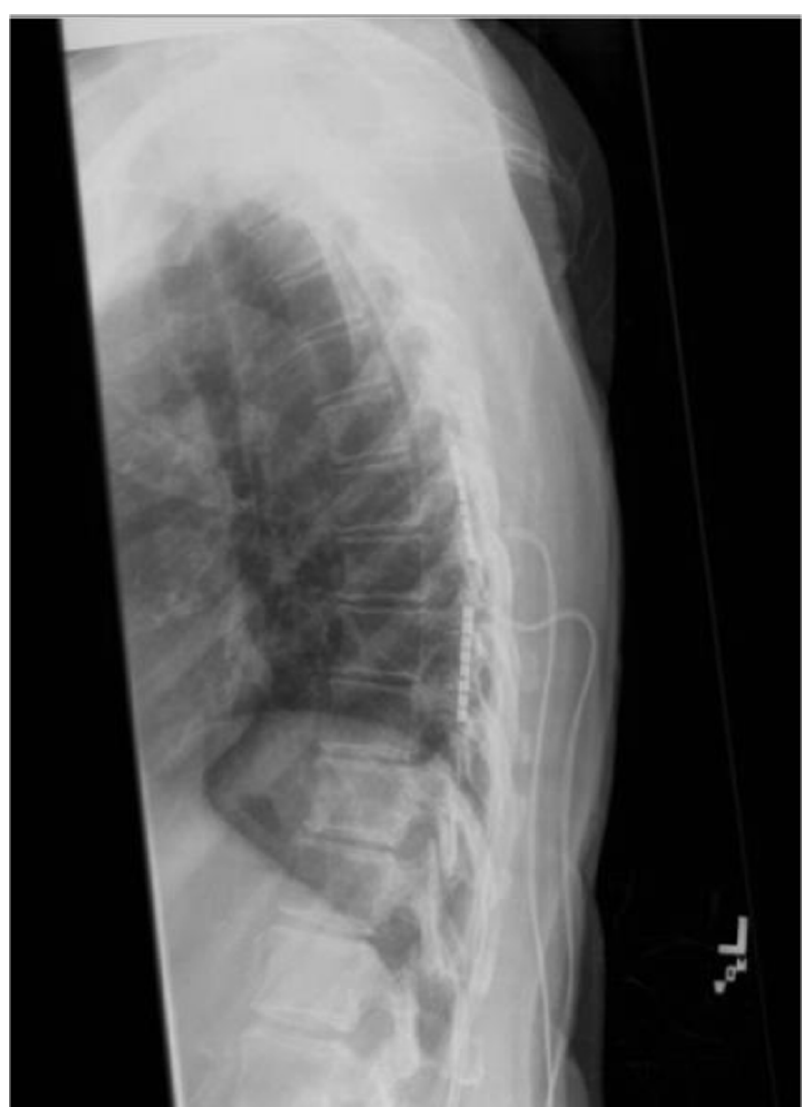

Figure $\mathbf{2}$ Lateral thoracic X-ray image showing permanent paddle lead placement in the thoracic interspaces. for this study since it was a retrospective analysis of a single case.

\section{Discussion}

We report the first case to our knowledge of a SCS being used successfully to treat multifactorial pain mediated by HMO. In this particular case, the pain was likely mediated by failed back syndrome, direct contact on the capsular joints, bone-mediated pain, and a mass effect on the surrounding soft tissue. Both the trial and the permanent implant phases of SCS were successfully able to reduce her overall pain intensity, improve her quality of life, and reduce her narcotic medication requirements.

Due to pain in multiple regions, this particular case illustrates the utility of being able to use a 16-contact lead compared to an eight-contact lead during a single trial. By spanning across 2.5 vertebral levels with closer contacts, we were theoretically allowed broader spinal cord coverage to better target the back, hip, and ankles. We would recommend that physicians who treat multiregional neoplastic pain with SCS consider this multilevel approach in order to accommodate 1) more dynamic pain patterns and 2) reduce the need for a staggered single lead coverage trial over multiple pain areas. ${ }^{12,13}$ However, further studies certainly are needed to prove whether this approach is more efficacious clinically and not just theoretical.

After performing a MEDLINE search, we were unable to identify any other cases of treating nonmalignant neoplastic bone pain with SCS. However, cancer-related neoplasms have been treated successfully in a manner similar to our case. ${ }^{11,14}$ Of note, Yakovlev and Resch reported using SCS to treat 15 patients with cancer-related back pain all of whom showed significant improvement. ${ }^{15}$ Additionally, there are several spinal metastasis case reports indicating improvement after similar treatment. ${ }^{16,17}$

In general, past review articles have rarely discussed SCS as an indication to treat neoplastic-mediated pain. ${ }^{18}$ This is likely due to the fact that no randomized controlled trials have been published concerning this scenario. Further research should be considered in treating neoplastic-mediated pain that has been resistant to both pharmacological and surgical modalities with SCS.

\section{Acknowledgments}

Departments of Anesthesiology and Perioperative Care, Medicine, Physical Medicine and Rehabilitation, and Neurosurgery contributed from University of California Irvine. 
Each department contributed physicians and resources who participated in the overall care of this patient.

\section{Disclosure}

EY Chang is the recipient of Rehabilitation Medicine Scientist Training Program fellowship (K12 HD001097) and Institute for Clinical and Translational Science (UL1 TR000153) faculty career development fellowship (KL-2). All authors declare that they have no other competing interests in this work.

\section{References}

1. Solomon L. Hereditary multiple exostosis. Am J Hum Genet. 1964;16: 351-363.

2. Schmale GA, Conrad EU, Raskind WH. The natural history of hereditary multiple exostoses. J Bone Joint Surg Am. 1994;76(7):986-992.

3. Legeai-Mallet L, Munnich A, Maroteaux P, Le Merrer M. Incomplete penetrance and expressivity skewing in hereditary multiple exostoses. Clin Genet. 1997;52:12-16.

4. Göçmen S, Topuz AK, Atabey C, Şimşek H, Keklikçi K, Rodop O. Peripheral nerve injuries due to osteochondromas: analysis of 20 cases and review of the literature. J Neurosurg. 2014;120(5):1105-1112.

5. Peterson HA. Multiple hereditary osteochondromata. Clin Orthop. 1989;239:222-230.

6. Kitsoulis P, Galani V, Stefanaki K, et al. Osteochondromas: review of the clinical, radiological and pathological features. In Vivo. 2008;22(5): 633-646.

7. Boswell MV, Trescot AM, Datta S, et al; American Society of Interventional Pain Physicians. Interventional techniques: evidence-based practice guidelines in the management of chronic spinal pain. Pain Physician. 2007;10(1):7-111.
8. Cameron T. Safety and efficacy of spinal cord stimulation for the treatment of chronic pain: a 20 -year literature review. $J$ Neurosurg. 2004;100(3 Suppl):254-267.

9. Linderoth B, Foreman RD. Physiology of spinal cord stimulation: review and update. Neuromodulation. 1999;2(3):150-164.

10. Oakley JC, Prager JP. Spinal cord stimulation: mechanisms of action. Spine (Phila Pa 1976). 2002;27(22):2574-2583.

11. Lihua P, Su M, Zejun Z, Ke W, Bennett MI. Spinal cord stimulation for cancer-related pain in adults. Cochrane Database Syst Rev. 2013;2: CD009389.

12. Falowski S, Celii A, Sharan A. Spinal cord stimulation: an update. Neurotherapeutics. 2008;5(1):86-99.

13. Perret D, Chang EY, Hata J, Chen H, Tribuzio B. The Pain Center Manual. New York: Demos Medical Publishing; 2013.

14. Shimoji K, Hokari T, Kano T, et al. Management of intractable pain with percutaneous epidural spinal cord stimulation: differences in pain-relieving effects among diseases and sites of pain. Anesth Analg. 1993;77(1):110-116.

15. Yakovlev AE, Resch BE. Spinal cord stimulation for cancer-related low back pain. Am J Hosp Palliat Care. 2012;29(2):93-97.

16. Tsubota S, Higaki N, Nagaro T. A case of neuropathic cancer pain in the lower extremities successfully treated with spinal cord stimulation. Masui. 2009;58(11):1460-1461.

17. Flagg A, McGreevy K, Williams K. Spinal cord stimulation in the treatment of cancer-related pain: "back to the origins". Curr Pain Headache Rep. 2012;16(4):343-349.

18. Song JJ, Popescu A, Bell RL. Present and potential use of spinal cord stimulation to control chronic pain. Pain Physician. 2014;17(3): 235-246.
Journal of Pain Research

\section{Publish your work in this journal}

The Journal of Pain Research is an international, peer-reviewed, open access, online journal that welcomes laboratory and clinical findings in the fields of pain research and the prevention and management of pain. Original research, reviews, symposium reports, hypothesis formation and commentaries are all considered for publication.

\section{Dovepress}

The manuscript management system is completely online and includes a very quick and fair peer-review system, which is all easy to use. Visit http://www.dovepress.com/testimonials.php to read real quotes from published authors. 